Musées, Patrimoine et Culture scientifiques et techniques

$138 \mid 2011$

novembre - décembre 2011

\title{
Les contaminations fongiques
}

Pest control on museum objects using heat-treatment

\section{Tony Basset et Caroline Laffont}

Édition électronique
URL : http://journals.openedition.org/ocim/994

DOI : 10.4000/ocim.994

ISSN : 2108-646X

Éditeur

OCIM

Édition imprimée

Date de publication : 1 novembre 2011

Pagination : 48-54

ISSN : 0994-1908

Référence électronique

Tony Basset et Caroline Laffont, "Les contaminations fongiques », La Lettre de I'OCIM [En ligne],

138 | 2011, mis en ligne le 01 novembre 2013, consulté le 19 avril 2019. URL : http://

journals.openedition.org/ocim/994; DOI : 10.4000/ocim.994 


\section{Les contaminations fongiques}

\section{Tony Basset et Caroline Laffont *}

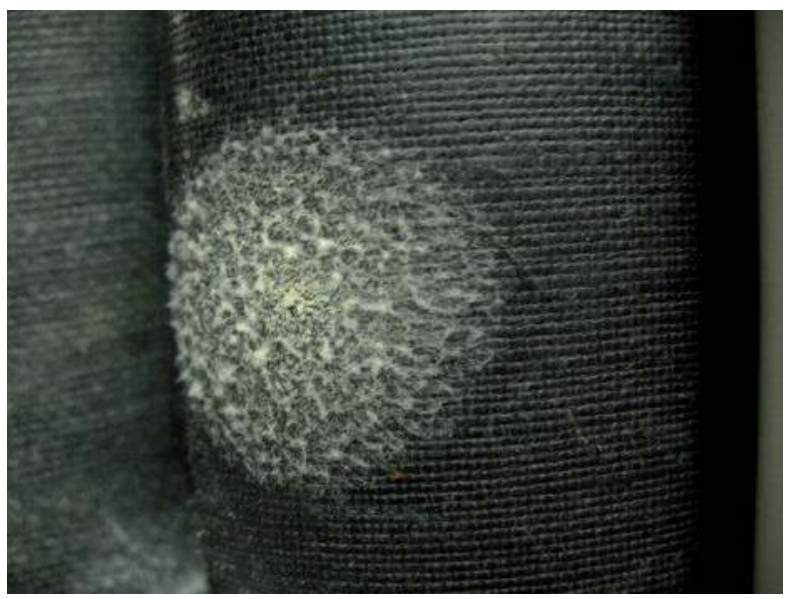

Présence de mycélium blanc sur la reliure d'un ouvrage () Bibliothèque nationale de France/Caroline Laffont

* Tony Basset est responsable des réserves du musée des Arts et Métiers tony.basset@cnam.fr Caroline Laffont est responsable du laboratoire de Microbiologie et de la Conservation préventive de la Bibliothèque nationale de France caroline.laffont@bnf.fr
Les auteurs font le point sur les mesures préventives et curatives pour la maîtrise du risque de biodéterioration dans les environnements patrimoniaux - mettant notamment en lumière la nécessité d'une approche globale des cas de contamination ainsi que sur les apports et l'évolution de la recherche scientifique dans ce domaine.

Les moisissures (champignons microscopiques), présentes dans tous les environnements, contribuent, avec d'autres micro-organismes, à la biodégradation et au recyclage des matières organiques. Leurs caractéristiques biologiques sont si intéressantes que certaines sont utilisées dans l'alimentation comme le Penicillium roquefortii pour la production de fromages, d'autres sont utilisées pour la production de médicaments comme la pénicilline produite par le Penicillium chrysogenum.

Les moisissures n'ont malheureusement pas que des effets bénéfiques. Elles peuvent aussi provoquer d'importantes détériorations notamment dans les domaines agronomiques, alimentaires et patrimoniaux.

En effet, dans un musée, le développement de moisissures engendre des dégradations qui peuvent se révéler irréversibles sur les matériaux organiques constitutifs des collections. Elles peuvent se concrétiser par l'apparition d'altérations inesthétiques sur les œuvres, comme conduire à la perte définitive du support de celles-ci.

En outre, la contamination d'une collection entraîne des nuisances de types différents certes, mais non moins importantes comme : l'immobilisation des collections, des risques sanitaires pour les agents et les visiteurs, et enfin un coût financier élevé généré par les opérations curatives lourdes. 


\section{La biologie des moisissures: les champignons inférieurs}

\section{Morphologie}

Les champignons constituent un groupe d'organismes d'une extrême variété allant des espèces microscopiques aux organismes macroscopiques. Le terme « moisissure » désigne tous les champignons microscopiques filamenteux présents dans l'environnement tant à l'extérieur qu'à l'intérieur. L'appareil végétatif appelé « thalle » n’est pas constitué de véritables tissus, il ne comporte ni racine, ni tige, ni feuille, à l'inverse des plantes. Ce thalle est constitué d'un réseau filamenteux appelé mycélium.

\section{Cycle de développement}

Le cycle de développement d'une moisissure comprend une phase végétative de croissance et de nutrition, et presque simultanément, une phase reproductive. La germination des spores est à l'origine de la phase végétative. Durant cette phase, la moisissure développe un réseau mycélien permettant la colonisation du support et la recherche de nutriments. Généralement, la présence de ces filaments sur les œuvres nous alerte de la potentialité d'une contamination.

Durant la phase reproductive, la moisissure va produire des particules de quelques microns, appelées « spores » qui assurent la dispersion de l'espèce. Ces spores, produites en très grande quantité, germent aussitôt si les conditions environnementales sont propices à cette germination. Ceci explique la propagation fulgurante d'une contamination. Par ailleurs, ces spores ont la particularité d'avoir un métabolisme réduit et d'être entourées de parois protectrices qui leurs confèrent une très grande résistance. Ainsi, elles peuvent rester inertes plusieurs années, attendant les conditions favorables pour leur germination et leur développement. Cet état s'appelle la « dormance » ou encore la «latence».

Dans les conditions ambiantes naturelles, les spores sont en suspension dans l'air, associées à des particules de plus grosses tailles que l'on nomme « poussière » (fibre animale, fibre végétale, textiles, squame...). Par sédimentation, ces spores se déposent sur toutes les surfaces : collection, rayonnage, sol, mur... Cette poussière est donc à la fois une réserve de spores et une source nutritive pour leur développement.

\section{Nutrition}

Ces microorganismes sont hétérotrophes, c'est-à-dire qu'ils se nourrissent de matière organique préexistante où ils puisent l'eau, les substances nutritives et les éléments minéraux nécessaires à leur croissance. Ce mode de nutrition s'oppose aux organismes autotrophes qui fabriquent leur matière organique à partir de la réduction de matière inorganique et

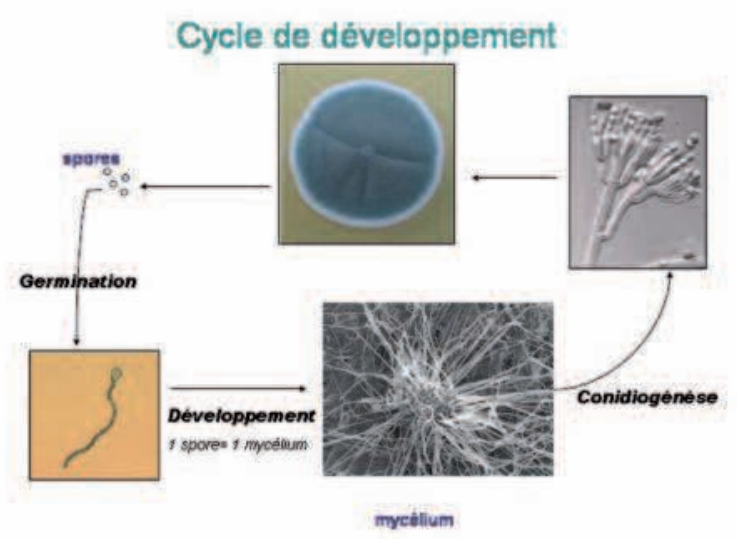

Cycle de développement des moisissures (c) Caroline Laffont

\section{Développement $=$ phase active}

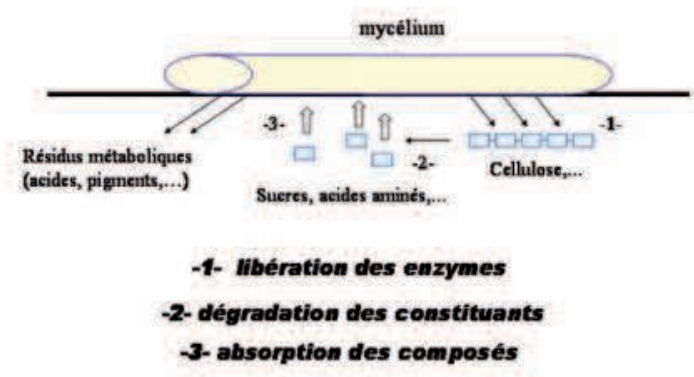

Mécanisme de dégradation d'un papier (c) Caroline Laffont et Tony Basset

d'une source d'énergie externe, comme les plantes qui utilisent l'énergie lumineuse et le gaz carbonique.

Par ailleurs, les moisissures néfastes pour les biens culturels, sont saprophytes, c'est-à-dire qu'elles se développent à la fois sur et au détriment des matériaux organiques inertes comme le cuir, les papiers, les bois, les aliments... Aussi, les retrouve-t-on fréquemment sur la plupart des supports organiques susceptibles d'être présents dans un musée. Ces supports organiques sont en général constitués de longues molécules (cellulose, gélatine, amidon, kératine, collagène...) que la moisissure est incapable d'ingérer telles quelles. La moisissure va donc excréter des enzymes, qui sont diffusées à la surface et à l'intérieur des matériaux favorisant ainsi la décomposition de ces longues molécules en petits éléments utilisés comme source nutritive. Les moisissures absorbent ensuite les produits organiques à travers la paroi de leur appareil végétatif, elles sont absorbotrophes.

La décomposition de ces longues molécules, première phase de la digestion du support, entraîne bien évidemment une détérioration des supports. En fin de digestion, la moisissure 
excrète ses déchets ; ce sont en général des acides, des pigments, qui eux aussi participent à la détérioration des matériaux.

\section{Les paramètres favorisant leur développement}

En plus de la disponibilité nutritive présente en grande quantité, le développement des champignons dépend de facteurs environnementaux dont les principaux sont l'eau et la température.

La quantité d'eau disponible dans le substrat et dans l'air environnant est le facteur principal pour initier leur développement. La phase de germination nécessite un apport d'eau plus important que la phase de croissance. Il est admis qu'à partir d'une humidité relative de $60 \%$, les spores peuvent se réhydrater naturellement, il y a donc un risque de germination. Une fois la germination enclenchée, le processus de développement peut se poursuivre à des taux d'humidité relative inférieurs à $60 \%$. La croissance de la moisissure ralentit si l'humidité relative baisse et s'arrête aux environs de $30 \%$ d'humidité relative, mais elle ne meurt pas, elle entre en phase de dormance et attend que les conditions redeviennent favorables pour germer.

La température n'est pas un facteur déclenchant comme l'eau. Ce paramètre influe uniquement sur la vitesse de croissance des moisissures. La plupart des champignons se développant sur les collections sont mésophiles ; c'està-dire qu'ils se développent autour de $20-25^{\circ} \mathrm{C}$ (température qui correspond à la moyenne habituelle des locaux de conservation). Une température inférieure à $20^{\circ} \mathrm{C}$ commence à ralentir la vitesse de croissance et à $0^{\circ} \mathrm{C}$ les réactions biochimiques sont presque toutes arrêtées. Cependant, le maintien d'une température négative, même si elle arrête la croissance et peut détruire les formes végétatives, n'éradique pas une contamination.

\section{Gestion d'une contamination fongique}

Si une contamination est suspectée sur des objets, une approche globale est nécessaire. Celle-ci va de la mise en place de mesures urgentes pour limiter la propagation à l'élaboration d'un plan d'action intégrant des mesures curatives et préventives pour la traiter de façon pérenne ${ }^{(1)}$.

\section{Les mesures urgentes}

Parmi les mesures urgentes, l'isolement des objets suspects, en les enveloppant dans du papier neutre de préférence, évite toute dissémination de la contamination. Ces objets sont à stocker dans un local de quarantaine, hors du magasin. Pour définir l'ampleur de la contamination, tous les objets stockés dans la même pièce que les objets suspects doivent être examinés et isolés s'ils présentent les mêmes symptômes. Dans les cas les plus graves, on peut être amené à isoler le local contaminé du reste du bâtiment, notamment par rapport aux gaines de circulation d'air qui seront obstruées si elles communiquent avec d'autres espaces.

En concomitance de ces actions, le risque sanitaire est à prendre en compte : les objets ne doivent plus être communiqués et des mesures doivent être prises afin de protéger les agents par le port d'équipements de protection individuelle.

Afin de garantir une meilleure gestion de la contamination, il est souvent indispensable de s'entourer de spécialistes privés ou institutionnels : scientifiques (Centre de Recherche sur la Conservation des Collections, Laboratoire de Recherche des Monuments historiques, Centre de Recherche et de Restauration des Musées de France, Centre interrégional de Conservation et Restauration du Patrimoine), restaurateurs, climaticiens.

Pour établir le plan d'action, des analyses microbiologiques doivent être réalisées afin d'identifier les souches développées sur les collections et évaluer leurs capacités de dégradation (2). Ces analyses permettent également de connaître l'état de vitalité des moisissures développées. De ces résultats découlera le choix du traitement curatif. Dans un deuxième temps, il faut rechercher les causes de la contamination et mettre en place des mesures correctives pour retrouver une situation normale. En effet, le départ d'une contamination est souvent précédé d'une élévation même ponctuelle de l'humidité relative, favorisant la germination des spores présentes dans tous les locaux de stockage. Ce déséquilibre climatique peut avoir plusieurs origines, comme par exemple une défaillance humaine, une défaillance du bâtiment (inondation, infiltration d'eau, arrêt du chauffage...) (3).

Ce n'est qu'à la lueur de ces différents résultats que les mesures correctives seront définies.

\section{Les mesures correctives}

Outre le retour à la normale des conditions climatiques, les mesures correctives englobent les traitements curatifs des collections et des locaux. Il est très difficile ici de définir une méthode unique, car le choix du traitement curatif, étape cruciale et difficile, dépend de plusieurs critères : l'étendue de la contamination (localisée ou généralisée à un magasin), la souche identifiée et sa virulence vis-à-vis des supports contaminés, les matériaux infestés, le type de collection contaminé (patrimoniale ou non), les moyens humains et financiers disponibles..

Par ailleurs, le traitement curatif doit aussi répondre à la déontologie de la conservation préventive, notamment concernant sa compatibilité avec les matériaux à traiter. Parallèlement à ce contexte, la législation sur l'emploi des produits chimiques a considérablement évolué, limitant le type de produit chimique encore utilisable. Ainsi face à 
ces différentes contraintes, nous n'établirons pas ici la liste des traitements chimiques ou physiques de désinfection susceptibles d'être utilisés pour les collections ou les locaux. Le choix du traitement ${ }^{(4)}$ est à faire en concertation avec les différents acteurs de la conservation : le conservateur, le personnel scientifique, le restaurateur.

Dans la mesure du possible, des traitements alternatifs, moins interventionnistes, sont mis en œuvre tant pour les collections que pour les locaux et le mobilier. Citons par exemple le dépoussiérage des objets moisis ${ }^{(5)}$ (6) pour des contaminations peu actives ou touchant très peu d'objets. Ce type de traitement doit être réalisé par du personnel formé et avec du matériel bien spécifique. C'est un traitement, non pas de désinfection mais de décontamination c'est-à-dire que les spores non aspirées sont encore viables. C'est pourquoi, ce traitement est accompagné obligatoirement d'une surveillance climatique rapprochée, notamment après la réintégration des collections dans le local de stockage.

Une désinfection est un traitement ponctuel ce qui signifie qu'un objet désinfecté pourra être re-contaminé ultérieurement, s'il est remis dans des conditions favorables au développement des moisissures. Traiter les collections sans traiter la ou les causes de la contamination est inutile.

La gestion d'une contamination est un processus long, souvent coûteux et lourd à mettre en œuvre, qui peut prendre plusieurs mois : recherche des prestataires et des

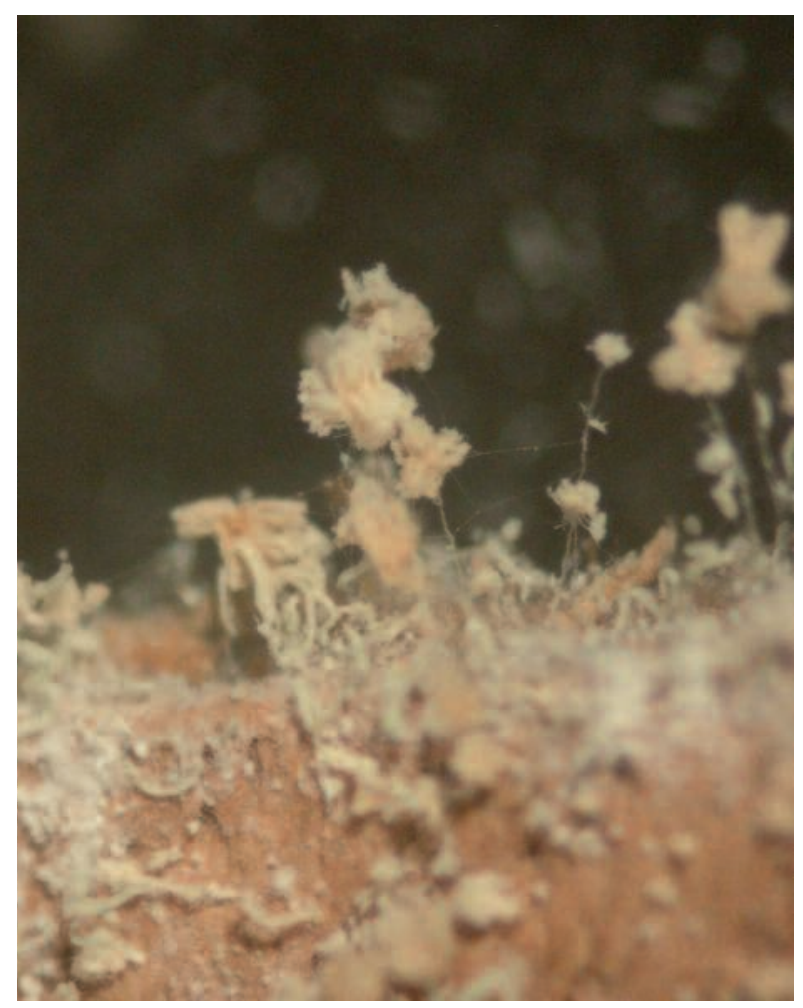

Ouvrage contaminé par un mycélium filamenteux. (c) Bibliothèque nationale de France/Stéphane Bouvet financements, réalisation des traitements... Il est donc d'un grand intérêt de mettre en place une politique de prévention de maîtrise de ces risques, intégrée à la politique de conservation du musée.

\section{Les moyens de prévention}

La conservation préventive est une démarche globale qui englobe l'ensemble des mesures prises pour prolonger la vie des objets, en prévenant, dans la mesure du possible, leur dégradation naturelle ou accidentelle (7). Tous les acteurs de la prévention s'accordent sur l'objectif principal qui n'est plus de procéder à des traitements curatifs des collections, mais de définir les meilleures conditions dans lesquelles les objets seront placés afin de limiter les risques de biocontaminations.

Ainsi, la prévention des contaminants biologiques est axée sur deux principes : diminuer l'apport des contaminants et éviter leur croissance.

\section{Prévenir l'apport des contaminants}

De par leur petite taille et leur légèreté, les spores de moisissures sont omniprésentes dans nos environnements. S'il est difficile de les éradiquer complètement d'un espace, on peut toutefois en limiter la quantité par une gestion raisonnée des collections et des locaux prenant en compte différents paramètres comme l'entretien, la filtration, l'entrée des collections en magasin avec des procédures de contrôle.

Ainsi, la filtration de l'air entrant dans un magasin apporte une garantie de la qualité de l'environnement. Ce principe est basé sur le passage de l'air entre des filtres particulaires qui arrêtent la majorité des particules de petite taille. Pour que ce système soit efficace et perdure dans le temps, il exige une maintenance régulière (changement des filtres) qui doit être contrôlée par les responsables de la conservation.

Les particules non retenues par ces filtres se déposent par sédimentation sur les collections et constituent une source de spores de moisissures ainsi qu'un substrat capable d'absorber l'humidité ambiante. La mise en place d'une surveillance microbiologique des collections, couplée à une surveillance microbiologique des surfaces et de l'air (8) est donc nécessaire pour le suivi régulier de la salubrité des collections. Cette surveillance permettra de mettre en place des procédures de nettoyage des locaux et des collections ${ }^{(9)}$.

Afin de limiter l'apport de contaminants extérieurs, les dons et les legs feront l'objet d'une attention particulière. Les responsables de la conservation préventive devront mettre en place un circuit d'entrée des nouveaux fonds (dons, legs), qui feront l'objet d'une mise en quarantaine et d'un nettoyage systématique avant leur stockage en magasin (10). 


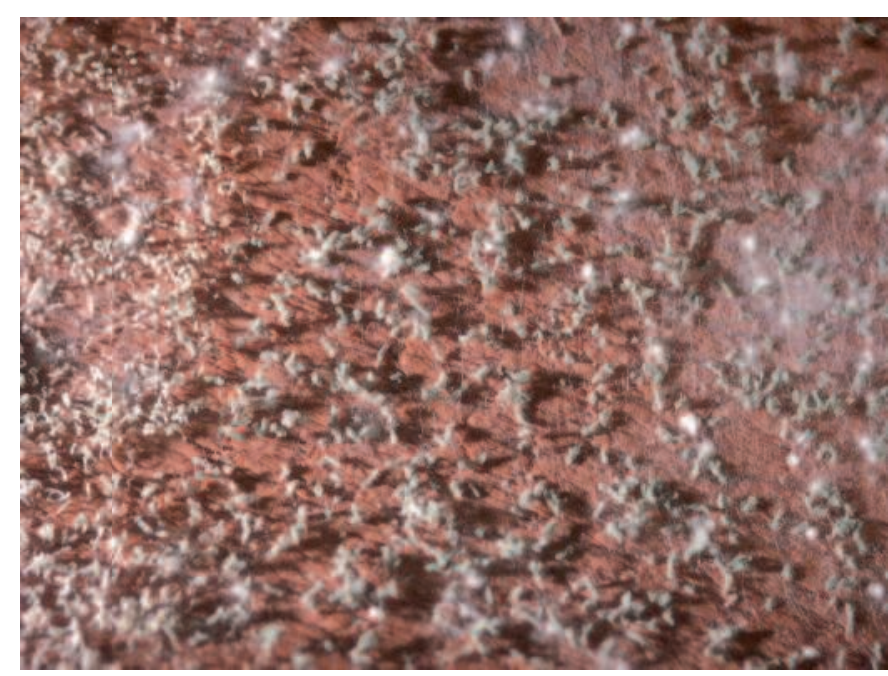

Surface de cuir contaminée par une moisissure. (C) Bibliothèque nationale de France/Stéphane Bouvet

Prévenir le développement d'une contamination Nous avons vu précédemment que la germination des spores de moisissures est sous l'influence des facteurs climatiques (température et humidité relative). Pour éviter le développement de ces contaminants, il est donc primordial de surveiller de façon permanente l'environnement climatique des collections ${ }^{(11)}$. Cette surveillance, réalisée grâce à des thermohygromètres électroniques ou mécaniques, permet d'intervenir quasi instantanément en cas d'élévation du taux d'humidité relative. Le taux d'humidité relative recommandé dans les locaux, se situe en dessous du seuil de germination des spores, soit 50-55 \% d'HR.

Par ailleurs, il est reconnu que dans un environnement clos ces deux facteurs climatiques ( $\mathrm{T}$ et HR) sont liés : toute hausse de température diminuant le taux d'humidité relative et toute baisse l'augmentant. Il est donc indispensable de contrôler ces deux paramètres en gardant pour objectif le maintien de la stabilité climatique avec une humidité relative aux alentours de $50 \%$ pour la plupart des matériaux et une plage de température de $18-20^{\circ} \mathrm{C}$. De surcroît, ces conditions sont aussi recommandées pour la prévention de l'ensemble des types de détériorations (physiques et chimiques).

Il est également nécessaire d'éviter les microclimats, c'est-àdire les zones où les conditions climatiques sont différentes des conditions ambiantes générales. Il peut s’agir de recoins où la circulation de l'air ne se fait pas bien, de rayonnages trop près de murs induisant un risque de condensation sur les objets. Ce risque peut être évité par une bonne ventilation assurant ainsi l'homogénéité du climat.

Afin de garantir un climat contrôlé, certains établissements ont été amenés à climatiser leurs espaces de stockage. La présence de telles installations implique une maintenance régulière et sérieuse car bon nombre de contaminations sont la conséquence d'installations mal entretenues ou en panne. Les responsables de la prévention doivent donc travailler de pair avec les équipes techniques. Le bâtiment lui-même, qui dans l'idéal doit être l'écrin protecteur garant d'une préservation à long terme des biens culturels, peut être défaillant : zones à risque pouvant exercer une action néfaste à la bonne conservation des collections, comme par exemple l'orientation des locaux (Nord/Sud), la présence de canalisations, de siphons, létat des huisseries : fenêtres, portes, murs (salpêtre, traces d'infiltration). Ainsi une bonne connaissance du bâtiment est nécessaire pour prévenir et corriger toutes anomalies. Par ailleurs, l'identification des problèmes liés au bâtiment permettra un stockage réfléchi des collections : les collections les plus sensibles dans l'endroit le plus stable et le plus sain par exemple.

\section{Évolution scientifique}

Une démarche de gestion du risque de biodégradation est un processus d'amélioration continu. La recherche scientifique (12) (13) (14) dont un bref résumé est présenté cidessous, contribue à améliorer et affiner cette démarche préventive.

\section{Assainissement de l'atmosphère des magasins} de stockage par le système mobile "Plasmair » Le système "Plasmair » (15), développé par la société Airinspace, est un système mobile permettant de maîtriser la contamination microbiologique de l'air dans l'industrie spatiale et dans le milieu hospitalier. La technologie employée repose sur la combinaison de champs électrostatiques multidirectionnels amplifiés et de plasma froid qui permet de détruire tous les microorganismes aéroportés. L'objectif de cette étude était de tester les performances de ce dispositif dans un magasin de conservation. Cette étude a été réalisée conjointement en 2007, dans les locaux des Archives nationales, par le Centre de Recherche sur la Conservation des Collections et par la Bibliothèque nationale de France.

Les résultats de cette étude ont démontré l'efficacité notable de ce système mobile d'assainissement en réduisant et en stabilisant la charge fongique en suspension dans l'atmosphère. Ce système peut donc être utilisé pour limiter les risques de contamination lors d'un sinistre ou pendant une campagne de dépoussiérage.

\section{Projet DECAGRAPH}

Le soutien du ministère de la Culture et de la Communication a permis de lancer en 2009 une étude sur la détection précoce des contaminants biologiques et chimiques appliquée au patrimoine (collections de bibliothèques et d'archives). 


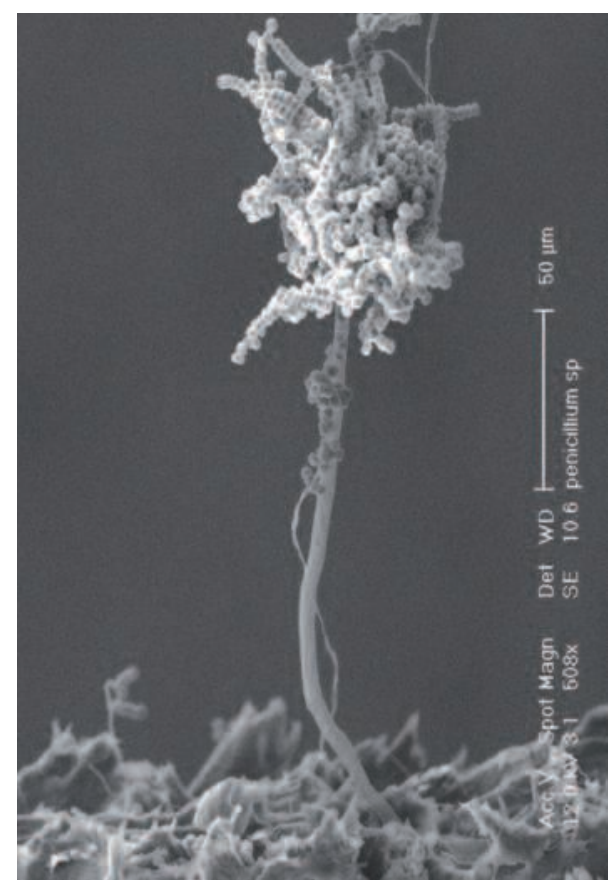

Surface d'un papier contaminé par une moisissure (pénicillium). () Bibliothèque nationale de France/Stéphane Bouvet

Cette étude - coordonnée par la Bibliothèque nationale de France, les Archives nationales, le Laboratoire de Recherche des Monuments historiques, le Centre scientifique et technique du Bâtiment et le Conservatoire national des Arts et Métiers - est le prolongement d'une précédente recherche menée par le Centre scientifique et technique du Bâtiment pour les lieux d'habitation et le Laboratoire de Recherche des Monuments historiques pour les Monuments historiques. L'originalité de cette recherche est basée sur l'analyse et la caractérisation des composés organiques volatils émis par les moisissures spécifiques des collections sur support papier, dès les premiers stades de développement $(\mathrm{COVm})$ c'est-à-dire la germination des spores de moisissures. L'objectif est de développer des capteurs chimiques autonomes qui permettront de surveiller en continu la qualité biologique de l'air des magasins de conservation et d'alerter en cas de dépassement des seuils nuisibles pour les collections patrimoniales. Les résultats de cette étude seront publiés courant 2012, mais les premiers résultats sont d'ores et déjà très prometteurs.

\section{Mycologie prévisionnelle}

La germination des spores de moisissure est un phénomène naturel, complexe sous l'influence de nombreux autres paramètres dont les principaux sont la température et l'humidité. L'objectif de la mycologie prévisionnelle (16) est de créer des outils mathématiques capables de prédire la germination des spores en tenant compte de l'ensemble des facteurs de l'environnement. Ainsi, pour établir de tels modèles mathématiques, la mycologie prévisionnelle étudie l'influence concomitante de l'ensemble des paramètres : conditions climatiques de l'air ambiant, mais aussi type de support et son activité en eau, présence d'inhibiteur interne, $\mathrm{pH}$, interactions entre les différents organismes qui se développent sur le même support (phénomènes de compétition pour le substrat et phénomènes de synergie), état physiologique des spores (stress subi, âge), conditions de production des spores.

L'usage d'un tel outil, s'il est développé, permettra de prédire la probabilité de germination et ainsi de mettre en place des mesures correctives avant même la détection visuelle d'un départ de contamination.

\section{Conclusion}

La prévention des biodétériorations est une approche globale et itérative qui s'insère dans la politique de conservation préventive de l'établissement. C'est une démarche quotidienne qui intègre autant la maîtrise du bâtiment dans sa conception et sa maintenance, que les procédures de gestion des entrées des collections, de manipulation et d'entretien. Elle nécessite des interventions priorisées pour la planification des investissements.

La réussite d'une telle démarche passe par la collaboration de tous les acteurs mais aussi nécessairement par la sensibilisation des agents aux contaminants biologiques et à leurs facteurs de développement.

\section{Notes}

(1) Laffont, C. Que faire face à une contamination biologique ? Actualités de la conservation, $\mathrm{n}^{\circ 24}$, 2005-2006, pp. 4-5. www.bnf.fr/pages/zNavigat/ frame/infopro.htm

(2) Bibliothèque nationale de France : www.bnf.fr/documents/evaluer_ contamination.pdf

(3) Basset, T. Preventive Preservation, a day to day work, International Preservation News, ${ }^{\circ} 41$, juillet 2007.

(4) Basset, T. Les traitements de désinfection du patrimoine écrit, Actualité de la conservation, $\mathrm{n}^{\circ} 24$, juillet 2005. www.bnf.fr/fr/professionnels/actualites_de_la_conservation

(5) Basset, T. et Simeone, L. Dépoussiérage des supports organiques moisis après une période de séchage, Preprints ICOM-CC triennal meeting de New Delhi 2008.

(6) Guild, S. et Macdonald, M. Prévention des moisissures et récupération des collections, lignes directrices pour les collections du patrimoine, Bulletin technique de l'ICC, n²6, 2004. www.cci-icc.gc.ca

(7) Vadémécum de la conservation préventive. Centre de Recherche et de Restauration des Musées de France. www.c2rmf.fr/documents/Vade_Mecum_ ConservPrev.pdf 
(8) Leclerc, B. Le contrôle de la biocontamination de l'air à la Bibliothèque nationale de France. Biodétérioration et désinfection des collections d'archi-

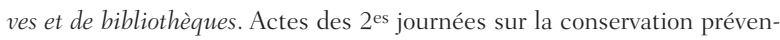
tive, Arles, 18 et 19 novembre 1996. Arles : Centre de Conservation du Livre, 1999. pp. 88-94.

(9). Basset, T., Genty, G. et Tallada, C. Étude comparative de quatre procédés de désinfection et de trois procédés de nettoyage sur quatre types de supports présents en bibliothèques. Actualités de la conservation, $\mathrm{n}^{\circ} 24$, 2005-2006, pp. 9-10. www.bnf.fr/fr/professionnels/actualites_de_la_ conservation

(10) Basset, T. Création d'une chaîne d'entrée des dons à la bibliothèque nationale de France, Actualité de la conservation, n²8, 2009. www.bnf.fr/ fr/professionnels/actualites_de_la_conservation

(11) Chardot, P. Le contrôle climatique dans les bibliothèques. Paris : Direction du Livre et de la Lecture, 1989

(12) www.crcc.cnrs.fr rubrique thème de recherche et projets de rechercher en coopération

(13) www.culture.gouv.fr/culture/conservation/fr/index.htm

(14). www.cicrp.fr/

(15). Rakotonirainy, S. et Basset, T. Assainissement de l'atmosphère dans les magasins de documents graphiques : évaluation des performances du système mobile « plasmair », Support tracé, nº 8, 2008, pp. 70-75.

(16) Dantigny, P. Preservation of books, pictures, fabrics and other from mould spoilage, International Preservation News, n4l, juillet 2007, pp. 19-21.

\section{Bibliographie}

Flieder, F. 1999. Sauvegarde des collections du patrimoine. Paris : CNRS Éditions.

Flieder, F. et Capderou, C. Sauvegarde des collections du Patrimoine : la lutte contre les détériorations biologiques. Paris : CNRS Éditions, 1999.

Laffont, C. et Mouren, R. Les ennemis du livre, Bulletin des bibliothèques de France, tome 50, ${ }^{\circ} 1,2004$, pp. 54-63. http://bbf.enssib.fr/

Patrimoine des bibliothèques, contamination des collections et des locaux des bibliothèques par des moisissures: méthodes de détection et d'évaluation. Paris : Direction du Livre et de la Lecture, 2001. www.culture.gouv.fr/culture/dll/contamination.pdf

Roquebert, M.-F. Les contaminants biologiques des biens culturels. Paris : Muséum national d'Histoire naturelle et Elsevier, 2002. 